\title{
EXPERIMENTAL AND NUMERICAL STUDY OF A NOVEL PIEZO- ELECTRIC PILOT STAGE FOR SERVOVALVES
}

\author{
Paolo Tamburrano ${ }^{1 *}$, Andrew R. Plummer ${ }^{2}$, Pietro De Palma ${ }^{1}$, Elia Distaso ${ }^{1}$ and Riccardo \\ Amirante ${ }^{1}$ \\ ${ }^{1}$ Department of Mechanics, Mathematics and Management (DMMM), Polytechnic University of Bari, Bari, Italy; \\ ${ }^{2}$ Centre for Power Transmission and Motion control (PTMC), University of Bath, Bath, UK. \\ *Corresponding author; e-mail address: paolo.tamburrano@poliba.it
}

\begin{abstract}
Two - stage servovalves, despite being widely used in aircraft and industry because of their reliability and high performance, have a few disadvantages that are still unsolved at the state of the art, such as the power consumption caused by the quiescent flow (internal leakage) in the pilot stage, and the complexity and high number of parts of the torque motor assembly of the pilot stage. The solution to these problems can help to reduce costs, weight, power consumption, and to enhance the reliability and producibility as well as the performance of these valves. This paper presents a novel configuration of servovalve, which has the potential to overcome the above-mentioned issues. The proposed servo-valve includes a novel architecture for the pilot stage by using two piezo-electric actuators (ring benders). In this paper, the performance of this novel pilot stage is assessed. To this end, a valve prototype has been constructed and tested; the experimental results are also used to validate a numerical model obtained with the software Simscape Fluids. The results show that, acting on specific parameters, the performance of the piezo-valve can be very competitive, while ensuring very low internal leakage and complexity.
\end{abstract}

Keywords: servovalves, internal leakage, piezoelectric actuators, ring bender

\section{INTRODUCTION}

The architectures of commercially available twostage servovalves, which have not substantially changed for many years, can provide high reliability and excellent dynamics [1, 2]. Regardless of the pilot stage, which can be a double flapper-nozzle pilot stage or a deflector jet pilot stage, the main stage is usually a spool valve in which the main spool is actuated by a differential pressure generated at its extremities by the pilot stage. In this way, very high actuation forces are generated, which results in a much faster response and higher shear forces than proportional valves using proportional solenoids to directly drive the spool [3-6]. These characteristics have led to widespread use of these servovalve designs, which are fundamental components in closed-loop electro-hydraulic motion control systems demanding high performance levels. However they present some week points that are still unsolved, such as: the quiescent flow in pilot stage (to be referred to as the internal leakage of the pilot stage), which is continuous and constant regardless of the opening degree of the main stage, thus causing unwanted power consumption during operation $[1,2]$, and the electromagnetic torque motor assembly which is composed of many sensitive mechanical and electrical parts that penalise simplicity, set-up, duration of manufacture and manufacturing costs $[1,2]$.

To date, research studies in the scientific literature have mainly been focused on the reduction of the complexity of servovalves. In particular, a promising research field aims to replace the electromagnetic torque motor assembly with piezoelectric actuators, thus reducing complexity and manufacturing costs [2]. Different types of piezoelectric actuators have been used to directly drive the main stage spool or to drive the flapper, jet pipe, or deflector jet in the pilot stage [7-16], showing that the idea of using a piezo-electric actuator to drive a servovalve is feasible and promising. Both piezostack actuators, in which several piezo elements are joined together to form a multi-layer actuator [7-9], and amplified piezo-stack actuators, which can provide higher displacement but lower forces [10-12], have been proposed to be used. 
However, both types seem to be too heavy and large to be employed in servovalves, which must be compact and light, especially for applications in aircraft. As an alternative to stack actuators, lighter piezo-actuators, such as rectangular benders, have been employed in novel designs to drive the pilot stage of servovalves in place of the torque motor [13-15]; in this case, the main deficit is the very low actuation forces obtained with these actuators.

Instead, the most promising solution for these applications seems to be "the ring bender", which is a flat annular piezo-disc deforming in a concave or convex fashion depending on the polarity of the applied voltage, providing a very good compromise between actuation force and displacement, while being sufficiently compact for this type of applications. In this regard, in [16, 17] two ring benders, mounted in tandem to provide redundancy, were used in place of the torque motor to drive the flapper in a double flapper-nozzle pilot stage. The preliminary results obtained in $[16,17]$ prove the feasibility, in terms of actuation forces, displacement and dimensions, of using ring benders as actuators for the pilot stages of servovalves.

All the above-mentioned research studies have had the objective of reducing the complexity of servovalves. However, to date the problem concerning the quiescent flow (internal leakage) in the pilot stage has not been addressed in the scientific literature, apart from the work presented in [18], in which a small spool actuated by a ring bender was employed for the pilot stage in order to minimise the internal leakage of the pilot stage.

In this scenario, the present research project aims at developing a novel configuration of piezo-valve capable of reducing both the complexity and the internal leakage of two stage servovalves. The novel valve concept is described in the following section.

\section{NOVEL SERVOVALVE CONCEPT}

Fig. 1 shows the proposed valve design. In place of the torque motor, flapper and flexure tube of typical servovalves [2], the proposed architecture includes two small piezo-valves actuated by ring benders. Each piezo-valve is a very simple twoway two-position $(2 / 2)$ valve that has the advantage of being normally closed, which means that the ring bender stops the oil flow when the spool is at null. This can represent a huge improvement in terms of power consumption compared to the typical configurations, since the quiescent flow (internal leakage in the pilot stage) is ideally null when the spool is at null. In addition, the torque motor with all its drawbacks (complexity and noise sensitiveness) is removed. The only drawback resulting from the proposed solution is that the mechanical feedback is no more possible, and only an electrical feedback can be used, like a linear variable differential transformer (LVDT).

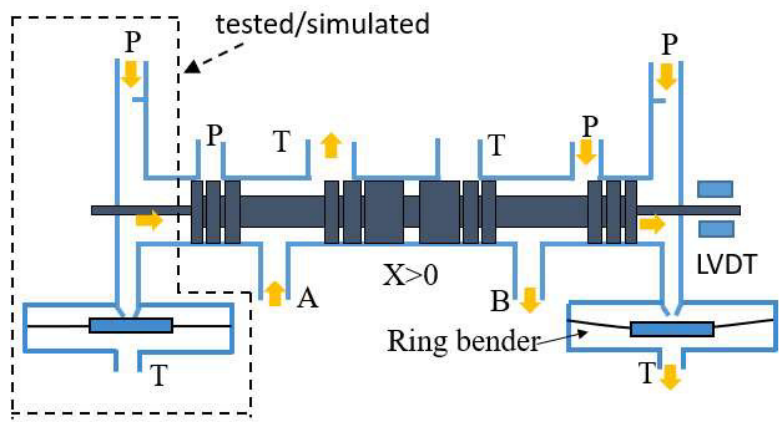

Figure 1: Operating principle of the novel servovalve pilot stage actuated by a ring bender

The feasibility of the proposed solution was already demonstrated in [19], in which a full simulation model of the full valve concept was used. The simulations showed that this valve architecture has a high potential in terms of response speed, in addition to minimising the internal leakage.

The feasibility study provided in [19] prompted the authors of this paper to construct a prototype of one of the two $2 / 2$ piezo-valves representing the novel pilot stage concept. The prototype was tested in a hydraulic test rig located at the Fluid Power Laboratory of the Centre of Power Transmission and Motion Control (PTMC) of the University of Bath. The prototype and the test rig are described in the next section.

\section{VALVE PROTOTYPE AND HYDRAULIC TEST RIG}

A prototype and a test rig were constructed and assembled in order to test one of the two piezovalves modulating the pressure at the main spool extremities. The test rig reproduces a part of the valve concept shown in Fig. 1 (see dashed box), being composed of the following components: supply line (P), fixed restriction, piezo-valve, and chamber comprised between the fixed restriction, 
the spool end and the piezo-valve. A schematic representation and a photograph of the test rig are shown in Fig. 2 and Fig. 3, respectively. The supply line is composed of a volumetric pump (1), a non-return valve (2), a pressure relief valve (3) and an accumulator (4). A pressure transducer (5) is positioned upstream of the restriction (6), whose area can be changed manually. This restriction represents the fixed restriction shown in Fig. 1, with the difference that, in the test rig, its flow area can be adjusted. The pressure drop across the restriction is measured by a second pressure transducer (7). After the restriction (6), the oil can enter the piezo-valve, whose components are positioned inside a case (8). An adjuster (9) was mounted inside the valve body through a nut so that the position of the nozzle (10) can be adjusted against the closure member (11). In this way, it is possible to provide the ring bender with a pre-compression to close the valve effectively. The closure member (11) was inserted through the hole of the ring bender (12), and the latter was fixed to the valve body using elastomers o-rings (13). This choice results from the fact that, in this way, the mechanical integrity of the ring bender is ensured while deforming inside the valve. The position of the closure member (therefore, the displacement of the ring bender) is measured through an eddy current sensor (14). Another adjustable mechanical stop (15) is also present in order to allow the maximum displacement of the ring bender (i.e., the maximum opening of the valve) to be adjusted. The pressure of the oil discharged from the piezo-valve is measured by a pressure transducer (16).

Note that the volume comprised between the restriction (6) and the nozzle (10) represents the volume of Fig. 1 comprised between the fixed restriction, the spool end and the nozzle of the piezo-valve.

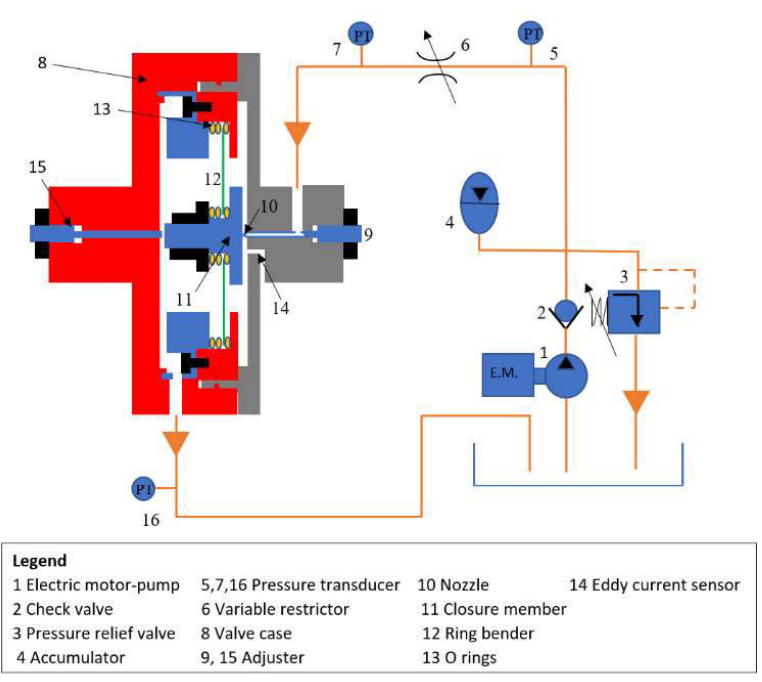

Fig. 2. Schematic representation of the test rig

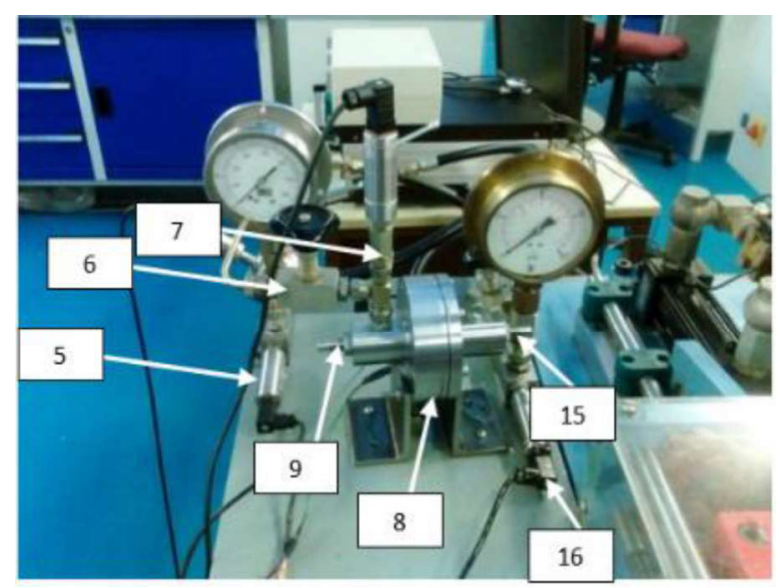

Fig. 3. Photograph of the test rig (see legend in Fig. 2 for comparison)

An external PC and a LabVIEW code were used to control the test rig and to acquire the signals from the transducers with a sample frequency of $10 \mathrm{kHz}$. The control signal was a control voltage (from $-5 \mathrm{~V}$ to $+5 \mathrm{~V}$ ) sent to the amplifier, which transformed this signal into a high voltage signal (from $-100 \mathrm{~V}$ to $+100 \mathrm{~V}$ ) applied to the ring bender. The pressure transducers are strain gages with a measurement error lower than $1 \%$ of the full scale (100 bar). Both the amplifier and the ring bender (model CMBR07) were purchased from the manufacturer Noliac [20]. The outer diameter and height of the ring bender are $40 \mathrm{~mm}$ $8 \mathrm{~mm}$, respectively. The ideal maximum displacement and force of the ring bender are \pm $185 \mu \mathrm{m}$ and $\pm 13 \mathrm{~N}$, respectively ( + denotes opening direction; - denotes closing direction). The tests were performed using ISO VG 32 hydraulic oil at a temperature of approx. $50{ }^{\circ} \mathrm{C}$ (oil density $=851 \mathrm{~kg} / \mathrm{m}^{3}$ and viscosity $=22.0 \mathrm{cSt}$ ). 
In the following, the same symbolism as that shown in Fig. 2 and Fig. 3 will be used to describe the numerical model and the experimental tests.

\section{NUMERICAL MODEL OF THE PIEZO- VALVE}

A numerical model simulating the test rig shown in Fig. 2 and Fig. 3, and hence the right part (or, equivalently, the left part) of the valve concept shown in Fig. 2, has been developed. The equations of the numerical model, which have been implemented into the Simscape Fluids environment [21], are described in the following.

The pump, pressure relief valve and accumulator are simulated through a "Hydraulic pressure source", which is an ideal source of hydraulic energy able to maintain a specific pressure at its outlet regardless of the flow rate consumed by the system. The value of the supply pressure is set equal to the value of the experimental tests, namely 71 bar.

The pressure drop from the supply line (4) to the pressure transducer (5), and across the variable restrictor (6), is simulated using the orifice equation:

$$
q=C_{D} A_{r} \sqrt{\frac{2 \Delta p}{\rho}}
$$

where $q$ is the flow rate, $\Delta p$ is the pressure drop and $\rho$ is the oil density $\left(\rho=851 \mathrm{~kg} / \mathrm{m}^{3}\right)$. The values of the equivalent restriction areas are tuned to match the experimental pressure drops, namely, $A_{r, 4-5}=4 \mathrm{~mm}^{2}$ and $A_{r, 6}=0.42 \mathrm{~mm}^{2}$ (with $C_{D, 4-5}=$ $C_{D, 6}=0.7$, under the hypothesis of turbulent flow).

The volume of oil comprised between the restriction (6) and the nozzle (10) is simulated using a block named "Constant Volume Hydraulic Chamber". With this block, a fixedvolume chamber with rigid walls is simulated and the fluid compressibility is taken into account. The following equations are applied:

$V_{\text {cham }}=V_{o}+\frac{V_{0} p}{E}$

$q_{c}=\frac{d V_{\text {cham }}}{d t}=\frac{V_{o}}{E} \frac{d p}{d t}$

where $V_{0}$ is the geometrical volume of the chamber (equal to the product of an internal diameter $D_{0}=20 \mathrm{~mm}$ and an overall internal length $L_{0}=60 \mathrm{~mm}$ ), $V_{\text {cham }}$ is the oil volume in the chamber at the pressure $p$, with $q_{c}$ denoting the flow rate through the chamber. The actual bulk modulus $E$ is corrected according to the relative gas content $\varepsilon$, which has been assumed equal to $\varepsilon=0.01$.

The hydraulic part of the piezo-valve is simulated using the orifice equation (1), in which the orifice area is calculated as $A_{r, v}=\pi d x$, where $d=1 \mathrm{~mm}$ is the diameter of the nozzle (10) and $x$ is the displacement of the ring bender (calculated with reference to the nozzle tip). The discharge coefficient of the piezo-valve is assumed equal to $C_{D, v}=0.65$ under the hypothesis of turbulent flow. The displacement $x$ of the ring bender is determined according to the equilibrium of the actuation force and the resistant forces acting on the ring bender, as follows:

$F_{r b}+F_{\text {flow }}-m_{0} \ddot{x}-C_{r b} \dot{x}-k_{r b}\left(x+x_{0}\right)=0$

where $F_{r b}$ denotes the force exerted by the ring bender having a stiffness $k_{r b}=70000 \mathrm{~N} / \mathrm{m}$ (data provided by the manufacturer); $C_{r b}$ is the damping coefficient of the moving parts (ring bender, o-rings and closure member) having equivalent mass $m_{0}=90 \mathrm{~kg} ; x_{0}=50 \mu \mathrm{m}$ is the pre-compression obtained through the adjuster (9), and $F_{\text {flow }}$ is the flow force acting on the ring bender. The damping coefficient was tuned to match the transient behaviour of the experimental displacement, thus obtaining $C_{r b}=26 \mathrm{Ns} / \mathrm{m}$.

Considering that the flow exiting the piezo valve can be assumed radial, the flow forces acting on the ring bender is calculated using equation (5):

$F_{\text {flow }}=p_{7} \frac{\Pi d^{2}}{4}$

The ring bender stroke is limited by a lower bound, which simulates the mechanical stop due to the presence of the nozzle (10). This stop is simulated as a spring and a damper having a spring stiffness $K_{\text {stop }}=10^{7} \mathrm{~N} / \mathrm{m}$ and a damping $C_{\text {stop }}=500 \mathrm{Ns} / \mathrm{m}$. These two parameters were tuned to obtain the same bouncing behaviour obtained experimentally when the closure member hits the mechanical stop.

Piezoelectric hysteresis is considered by implementing the Bouc-Wen hysteresis model, described and used in [19]. The Bouc-Wen model is represented by an equation [19] which allows the calculation of the hysteresis nonlinear term $n$. The hysteresis non-linear term allows the force of 
the ring bender $F_{r b}$ to be expressed as a function of the output voltage from the amplifier, as follows:

$F_{r b}=K_{d, v}\left(V_{a m p}-n\right)$

where $K_{d, v}=0.13 \mathrm{~N} / \mathrm{V}$ (data provided by the manufacturer) is the ring bender maximum force divided by the maximum operating voltage, which represents the ring bender conversion factor (namely, from amplified voltage to force).

The amplifier is simulated by using a second order transfer function:

$V_{a m p}=\frac{K_{a} \omega_{n}{ }^{2}}{s^{2}+2 \xi \omega_{n} s+\omega_{n}{ }^{2}} V_{c}$

where $V_{c}$ is the control voltage that is supplied to the amplifier (from $-5 \mathrm{~V}$ to $+5 \mathrm{~V}$ ), and $K_{a}$ is the gain of the amplifier $\left(K_{a}=20\right)$. In addition, to model the current limit $\left(I_{\max }=1 \mathrm{~A}\right)$, the rate of change of the amplified voltage is limited according to the following equation:

$\frac{d V_{a m p}}{d t}=\frac{I_{\max }}{C}$

where $C=3480 \mathrm{nF}$ is the capacitance.

The unknown parameters of the amplifier (natural frequency $\omega_{n}=1400 \mathrm{rad} / \mathrm{s}$ and damping factor $\xi=1.5$ ) have been set to obtain the same transient as that of the measured amplified voltage.

\section{RESULTS}

\subsection{Experimental results and comparison with the numerical model}

Step tests were performed on the test rig shown in Fig. 2 and Fig. 3. The supply (absolute) pressure was kept equal to 71 bar (namely, 70 bar relative), and the absolute pressure at the outlet of the valve was around 1 bar, with the oil temperature being kept around $50{ }^{\circ} \mathrm{C}$. These tests consisted in sending a step voltage to the amplifier, which, in turn, sent a corresponding amplified step voltage to the ring bender. The resulting displacement of the ring bender was measured along with the pressure at points 5 and 7 (see Fig. 2). The experimental results of these step tests are here presented and compared with the corresponding simulated step tests obtained with the numerical model described in Section 4.

In these step tests, the ring bender was precompressed with $x_{0}=50 \mu \mathrm{m}$ by acting on the adjuster (9); instead, the other adjuster (15) was kept far from the closure member, so that no mechanical stop was imposed to the maximum opening of the ring bender (11).

In the first test considered in this analysis, the control voltage (input voltage from the $\mathrm{PC}$ to the amplifier) was changed from $-5 \mathrm{~V}$ to $+5 \mathrm{~V}$, which produced an amplified voltage step from $-100 \mathrm{~V}$ to $+100 \mathrm{~V}$. Figure 4 (a) shows the control signal, and the amplified voltage measured and simulated during this test.

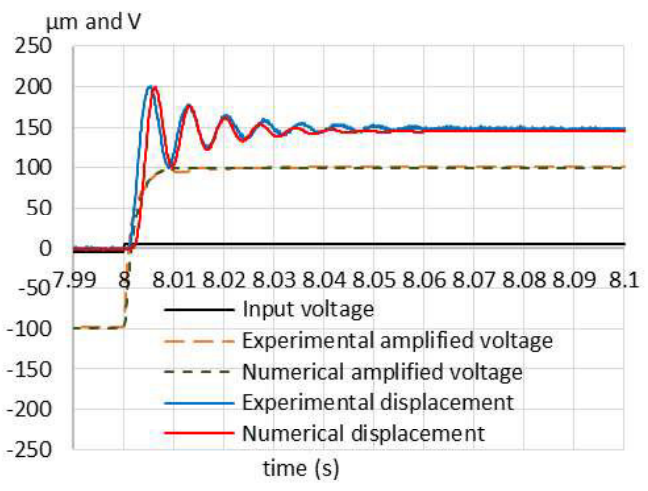

(a)

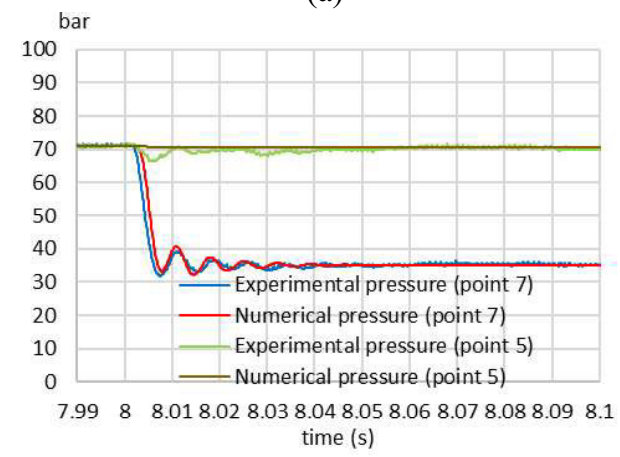

(b)

Fig. 4. Comparison between measured amplified voltage and simulated one and between measured displacement and simulated one for a positive step control voltage from $-5 \mathrm{~V}$ to $+5 \mathrm{~V}$ : (a); comparison between measured pressures and simulated ones: (b).

The good correspondence between the experimental amplified voltage and the numerical one demonstrates the good accuracy achieved with the numerical model and the good choice of the parameters used for the simulation of the amplifier.

Fig. 4 (a) also shows the displacement of the ring bender experimentally measured and simulated during the same step test. When $-100 \mathrm{~V}$ was applied to the ring bender, the latter applied a blocking force of about $-13 \mathrm{~N}$ to the nozzle (10) without moving (because it was pre-compressed). In contrast, when $+100 \mathrm{~V}$ was applied, the ring 
bender moved from the nozzle, thus opening the valve with a force of about $+13 \mathrm{~N}$. It can be noted that the simulated displacement matches the experimental one; this very good superimposition confirms the reliability of the numerical model. It is also revealed from the graph of Fig. 4 (a) that, because there is not a mechanical stop in the opening direction, the ring bender oscillates producing overshoots and undershoots until reaching a stable position. A further consideration that can be done is that the risetime is very fast, with less than $5 \mathrm{~ms}$ being needed to reach $90 \%$ of the maximum opening.

With reference to the same test, Fig. 4 (b) shows the comparison among the experimental pressures at points (5) and (7) and the corresponding numerical predictions. Also in this case, the comparison between the experimental data and numerical predictions is very good, especially regarding the pressure at point (7). With regard to the pressure at point (5), the numerical model is not able to reproduce the oscillations present in the experimental signal, which might be due to inertia effects of the oil comprised between the supply line and point (5). However, what is important in this analysis is the good prediction of the pressure at port (7), because this pressure signal is the one to be used for the control of a main spool.

As shown by the graph of Fig. 4 (b), the initial pressures measured at point (5) and point (7) were the same, namely, 71 bar, because the valve was initially closed by the ring bender. When the ring bender opened the valve, the pressure at port (7) decreased down very quickly to about a half of the supply pressure (this pressure drop was achieved by adjusting the variable restrictor (6)), reaching $90 \%$ of the minimum value within $5 \mathrm{~ms}$.

A second step test is now described; this test is the continuation of the previous one, in that, after reaching the maximum opening shown in Fig. 4, the valve was closed by changing the input signal to the amplifier from $+5 \mathrm{~V}$ to $-5 \mathrm{~V}$. The amplified voltages are reported in Fig 5 (a). Also in this case, there is a good correspondence between the experimental measurement and the numerical prediction, even though a slight delay is noticed in the experimental signal when the amplified voltage is approaching the minimum value $(-100$ V). However, this effect can be considered negligible since the experimental voltage and the numerical one reach a very high level of amplification (i.e., about $-90 \mathrm{~V}$ ) at the same time, which corresponds to a very high level of force (namely, about $-12 \mathrm{~N}$ ).

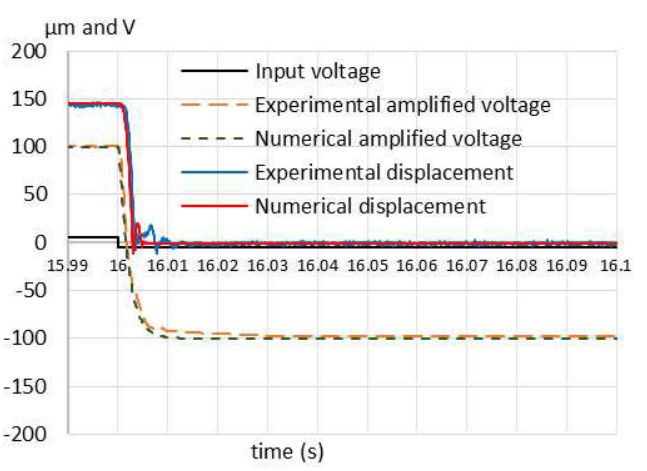

(a)

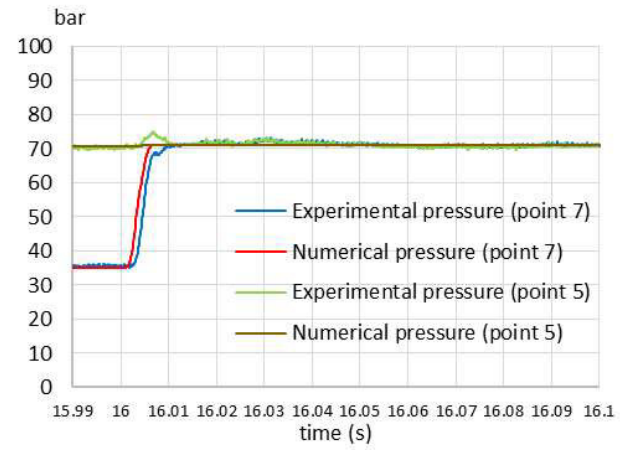

(b)

Fig. 5. Comparison between measured amplified voltage and simulated one and between measured displacement and simulated one for a negative step control voltage from $+5 \mathrm{~V}$ to $-5 \mathrm{~V}$ : (a); comparison between measured pressures and simulated ones: (b).

With reference to this second test, Fig. 5 (a) also shows the comparison between the experimental displacement and the numerical one: this graph again confirms the reliability of the numerical model, given the very good superimposition between the two curves. Moreover, the very good performance of the valve is highlighted by the very short time taken by the ring bender to close the valve (less than $5 \mathrm{~ms}$ ). In this case, no oscillations are present, because the nozzle (10) acts as a mechanical stop for the ring bender.

Fig. 5 (b) shows the pressure trends measured and predicted at points (5) and (7) in this second test. In addition to noticing, once again, the good accuracy of the numerical model, one could notice that the ring bender perfectly achieves its task, namely, it fully closes the valve, and the pressure at point (7) is brought back to its initial value of 71 bar very quickly. 


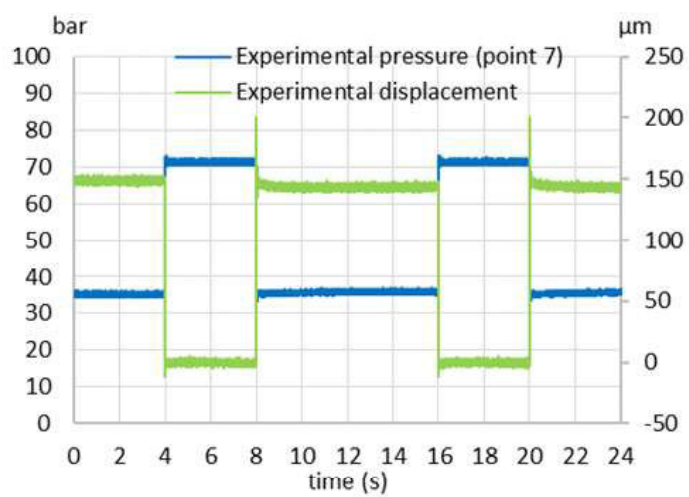

Figure 6. Pressure at point (7) and displacement measured during repeated opening and closing procedures of the piezo-valve.

Finally, Figure 6 shows, on the same graph, the displacement and the pressure at point (7) measured during repeated opening and closing procedures of the valve. This graph is instrumental in pointing out that, when the valve is closed, the pressure at point (7) is maintained constant over time and is equal to the supply pressure (71 bar). This means that the leakage through the valve is very low (almost null), thus achieving the main goal of this project, which is the drastic reduction of the internal leakage.

\subsection{Numerical analysis of the piezo-valve}

After having validated the numerical model, a numerical analysis is provided in this section to prove that, by properly acting on some geometrical parameters, the valve concept can be profitably applied for the control of a main stage spool valve.

At first, the effects of the volume of the chamber comprised between the restrictor (6) and the nozzle (10) are assessed. To assess the effects of reducing this geometrical volume, this analysis considers five volumes, having diameters $D=K$ $D_{0}$ and lengths $L=K L_{0}\left(D_{0}=20 \mathrm{~mm} L_{0}=60 \mathrm{~mm}\right)$ with $K=1,0.8,0.6,0.4,0.2$. The five cases are here compared in terms of simulated displacement of the ring bender and pressure at point (7), since these parameters are representative of the valve performance. In this regard, Fig. 7 (a) shows the ring bender displacement simulated for an amplified step voltage from $-100 \mathrm{~V}$ to $+100 \mathrm{~V}$ and supply pressure $=71$ bar. It is noteworthy that the reduction of the volume causes a reduction of the oscillations.

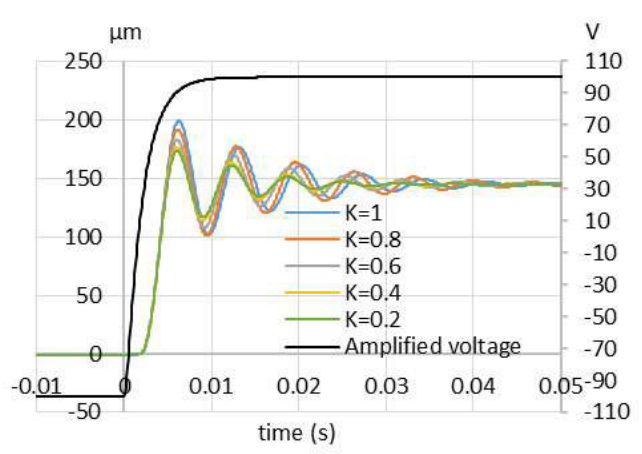

(a)

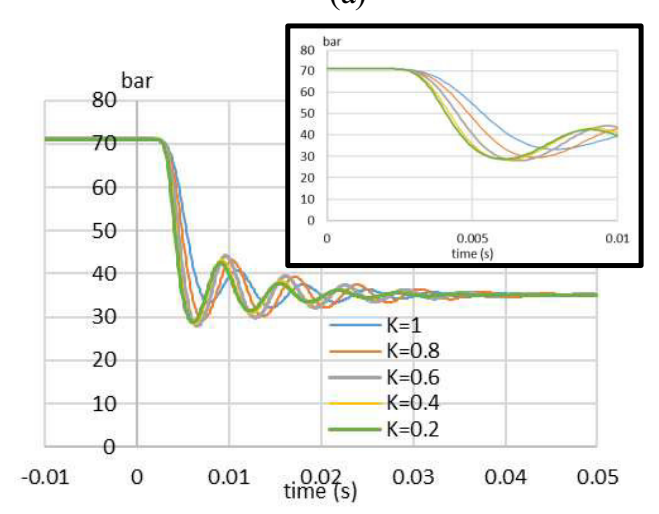

(b)

Fig. 7. Ring bender displacement (a) and pressure at point 7 (b), simulated for an amplified voltage from $-100 \mathrm{~V}$ to $+100 \mathrm{~V}$ and for $D=K D_{0}$ and $L=K L_{0}$, with $K=1,0.8,0.6,0.4,0.2$

The effects of the volume reduction are also important in terms of the pressure drop at point (7); as shown in Fig. 7 (b), smaller oil volumes cause the pressure to drop more quickly, as a consequence of the fact that a smaller oil volume can be discharged from the nozzle (10). This is particularly important for the actuation of a main spool: by reducing the oil volume in the system, the pressure at the spool ends will change more quickly, and the performance of the valve will be enhanced.

Similarly, Fig. 8 shows the ring bender displacement simulated for the five volumes, but, in this case, for an amplified voltage step from $+100 \mathrm{~V}$ to $-100 \mathrm{~V}$. In contrast to the previous case (voltage from $-100 \mathrm{~V}$ to $+100 \mathrm{~V}$ ), in this case the simulated trends of the displacement are almost identical, which means that the change in the volume does not affect the displacement.

However, as shown in Fig. 8 (b), the volume has a remarkable effect on the pressure rise at point (7), because lower volumes cause the compressibility effects to be less important. For this reason, it is important to reduce the volume of the chamber comprised between point (6) and 
the valve nozzle (10) to improve the performance of the valve. However, for $K=0.2$ and $K=0.4$, the simulated trends of the pressures are very similar, which means that a further reduction of the volume will not much change the results.

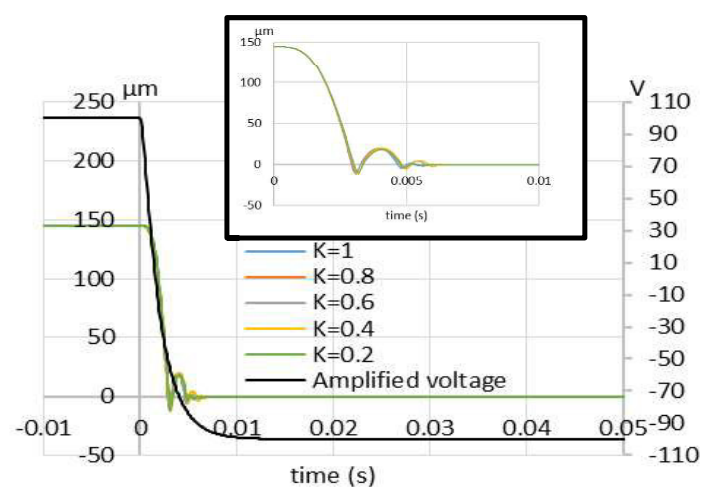

(a)

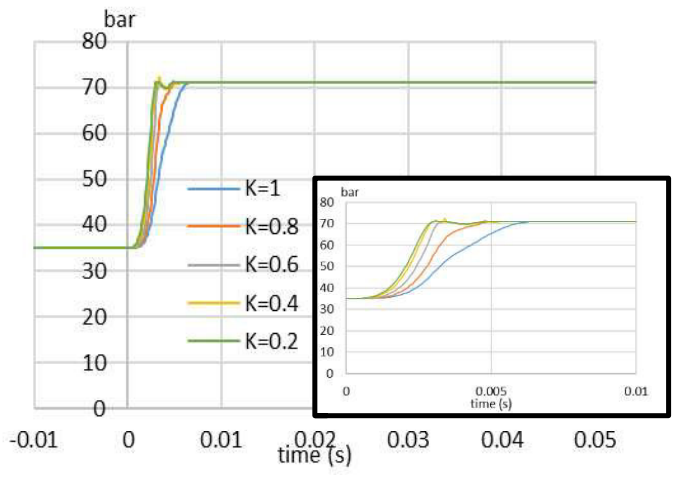

(b)

Fig. 8. Ring bender displacement (a) and pressure at point 7 (b), simulated for an amplified voltage from $+100 \mathrm{~V}$ to $-100 \mathrm{~V}$ and for $D=K D_{0}$ and $L=K L_{0}$, with $K=1,0.8,0.6,0.4,0.2$

Another parameter that can be optimised to further improve the performance of the piezovalve is the mass of the moving parts. To assess the effects of this parameter, this analysis considers five values for the mass, namely, $m=K$ $m_{0}$, with $K=1,0.8,0.6,0.4,0.2$ (with $m_{0}$ denoting the mass of the prototype). The diameter and length of the volume comprised between the restriction (6) and the nozzle (10) are taken equal to $D=0.6 D_{0}$ and $L=0.6 L_{0}$. The five cases are again compared in terms of simulated displacement of the ring bender and pressure at point (7). Fig. 9 (a) shows the different trends of the displacement for an amplified step voltage from $-100 \mathrm{~V}$ to $+100 \mathrm{~V}$. It is notable that the oscillations around the set point are remarkably reduced by reducing the mass of the moving parts; as a result, a lower mass allows the maximum displacement to be stably reached in shorter times.

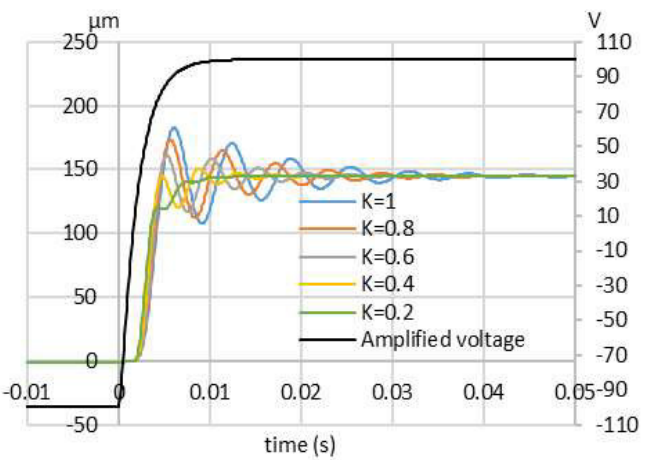

(a)

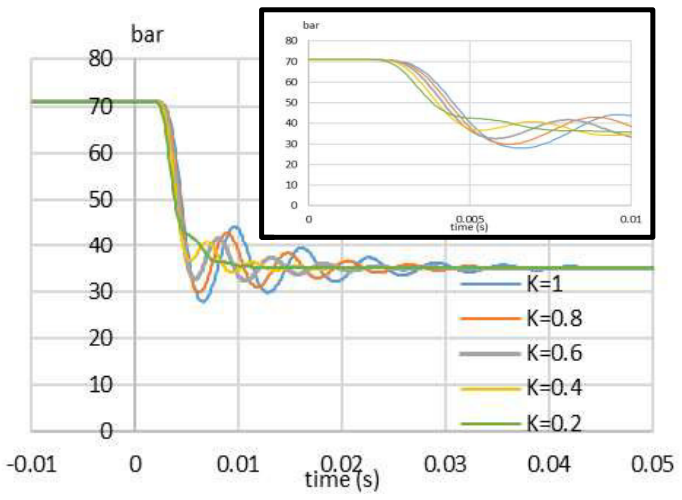

(b)

Fig. 9. Ring bender displacement (a) and pressure at point 7 (b), simulated for an amplified voltage from $-100 \mathrm{~V}$ to $+100 \mathrm{~V}$ and for different values of the mass of the moving parts: $m=K m_{0}$, with $K=1,0.8,0.6,0.4,0.2$ (supply pressure $=71 \mathrm{bar}$, $D=0.6 D_{0}$ and $\left.L=0.6 L_{0}\right)$

The beneficial effect of reducing the mass of the moving parts is also evident by analysing the simulated pressure drop at point 7 , as shown in Fig. 9 (b). It can be noticed that the lower values of the mass make the pressure reach a stable value more quickly than higher values of the mass, in addition to providing a slightly faster response due to the lower inertia.

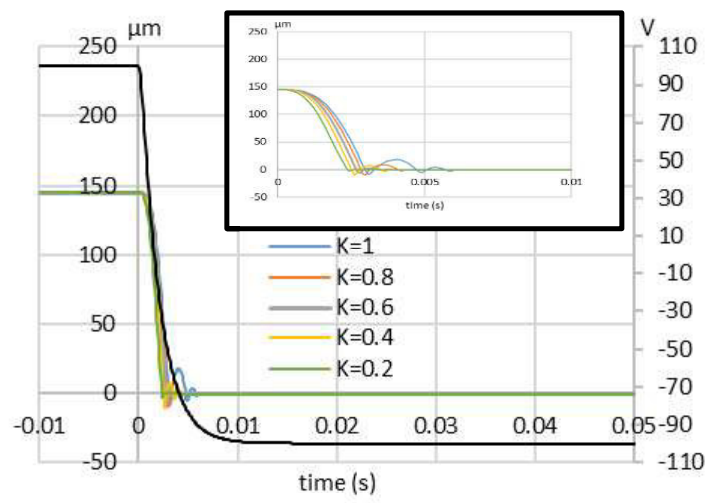

(a) 


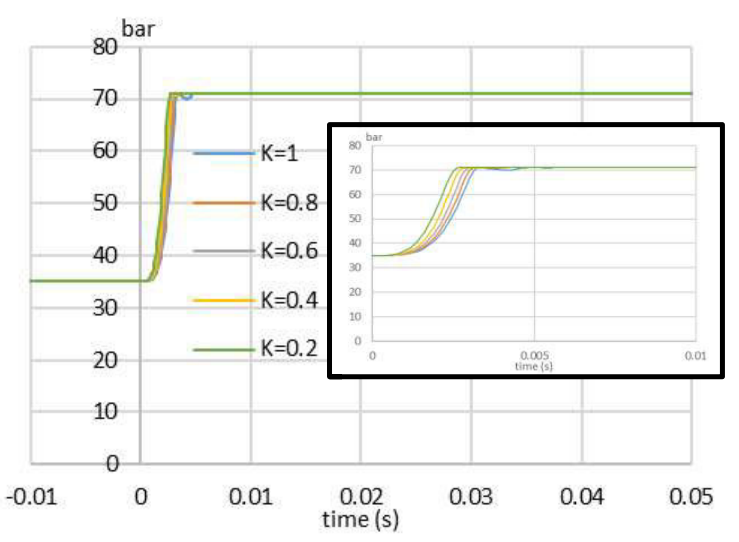

(b)

Fig. 10. Ring bender displacement (a) and pressure at point 7 (b), simulated for an amplified voltage from $+100 \mathrm{~V}$ to $-100 \mathrm{~V}$ and for different values of the mass of the moving parts: $m=K m_{0}$, with $K=1,0.8,0.6,0.4,0.2$ (supply pressure $=71 \mathrm{bar}$, $D=0.6 D_{0}$ and $\left.L=0.6 L_{0}\right)$.

Similarly, Fig. 10 shows the ring bender displacement simulated for $m=K m_{0}$, with $K=1$, $0.8,0.6,0.4,0.2$, but, in this case, for an amplified voltage step from $+100 \mathrm{~V}$ to $-100 \mathrm{~V}$. A difference is noticed in the final part of these curves. In fact, a lower mass causes the ring bender to bounce back with lower intensity after hitting the nozzle, in addition to providing a slightly faster response due to the lower inertia. Also in terms of pressure rise at point (7), as shown in Figure 10 (b), lower values of the mass cause the pressure at point (7) to increase slightly faster.

Overall, this numerical analysis has shown that the valve performance can be enhanced by reducing the volume of oil in the system and by reducing the mass of the moving parts. In these conditions, the response time of the valve is very fast, with the response time to reach $90 \%$ of the final value being lower than $5 \mathrm{~ms}$. This response time is comparable with commercially available pilot stages, but with the advantage of having eliminated the quiescent flow, which can be as high as $11 /$ min for commercially available units [17].

\section{CONCLUSION AND OUTLOOK}

This paper has proposed a novel architecture for the pilot stage of two stage servovalves, composed of two $2 / 2$ piezo-valves actuated by ring benders, which are compact elements providing good displacement and actuation forces. This novel configuration has the potential to overcome two main issues occurring in servovalves, namely, the complexity and the internal leakage of the pilot stage. To assess the validity of the proposed solution, an experimental prototype of the $2 / 2$ piezo-valve was constructed and experimentally tested in a hydraulic test rig. The step tests performed on the test rig show that the piezo-valve has a high potential in terms of response speed in addition to drastically reducing the internal leakage. The experimental data have been used to validate a numerical model obtained with Simscape Fluids. This model has been used to show that, by acting on some crucial parameters, such as the volume of oil in the system and the mass of the moving parts, the performance of the valve can be further improved.

\section{REFERENCES}

[1]. A. Plummer, "Electrohydraulic servovalves past, present, and future," 10th Int. Fluid Power Conf., pp. 405-424, 2016.

[2]. Tamburrano, P., Plummer, A. R., Distaso, E., \& Amirante, R. (2019). A review of electrohydraulic servovalve research and development. International Journal of Fluid Power, Volume 20, Issue 1, 2019, Pages 53-98.

[3]. Tamburrano, P., Plummer, A. R., Distaso, E., \& Amirante, R. (2019). A review of direct drive proportional electrohydraulic spool valves: industrial state-of-the-art and research advancements. Journal of Dynamic Systems, Measurement, and Control, 141(2), 020801.

[4]. Amirante, R., Distaso, E., \& Tamburrano, P. (2016). Sliding spool design for reducing the actuation forces in direct operated proportional directional valves: Experimental validation. Energy Conversion and Management, 119, pp. 399-410

[5]. Amirante, R., Distaso, E., \& Tamburrano, P. (2014). Experimental and numerical analysis of cavitation in hydraulic proportional directional valves. Energy Conversion and Management, 87, pp. 208-219

[6]. Amirante, R., Catalano, L. A., Poloni, C., \& Tamburrano, P. (2014). Fluid-dynamic design optimization of hydraulic proportional directional valves. Engineering Optimization, 46(10), pp. 1295-1314.

[7]. Bang, Y.B., et al. (2003). Development of a twostage high speed electrohydraulic servovalve systems using stacktype piezoelectric elements. 
In IEEE/ASME international conference on advanced intelligent mechatronics, AIM (Vol.1, pp. 131-136). Kobe, Japan: IEEE. doi:10.1109/AIM.2003.1225084J.

[8]. Branson, D.T., et al., 2011. Piezoelectrically actuated hydraulic valve design for high bandwidth and flow performance. Proceedings of the Institution of Mechanical Engineers. Part I: Journal of Systems and Control Engineering, 225 (3), 345-359. doi:10.1177/09596518JSCE1037

[9]. Reichert, M., 2006. High response hydraulic servovalve with piezo-actuators in the pilot stage. Olhydraulik and Pneumatik, 12, 1-17.

[10]. Lindler, J.E. and Anderson, E.H. (2002). Piezoelectric direct drive servovalve. In SPIE's 9th annual international symposium on smart structures and materials, 2002, San Diego, California, United States. doi:10.1044/10590889(2002/er01)

[11]. Jeon, C. Han, Y. M. Han, and S. B. Choi, “A new type of a direct-drive valve system driven by a piezostack actuator and sliding spool," Smart Mater. Struct., vol. 23, no. 7, 2014.

[12]. S. Karunanidhi and M. Singaperumal, "Mathematical modelling and experimental characterization of a high dynamic servo valve integrated with piezoelectric actuator," Proc. Inst. Mech. Eng. Part I J. Syst. Control Eng., vol. 224, no. 4, pp. 419-435, 2010.

[13]. Milecki A. Modelling and investigation of electrohydraulic servovalve with piezo element. Proc Inst Mech Technol 2006; 26: 181-188.

[14]. Zhu, L., et al. (2010). Development of hydroelectric servovalve based on piezoelectric elements. In 2010 Int. conf. mech. autom. control eng. MACE2010 (pp. 3330-3333).

[15]. Cheng, G.M., et al., 2005. Double-nozzle piezoelectric servovalve. Guangxue Jingmi Gongcheng/Optics and Precision Engineering, 13 (3), 276-282.

[16]. Bertin, M. J. F., Plummer, A. R., Bowen, C. R., Johnston, D. N., \& City, S. (2014, September). An investigation of piezoelectric ring benders and their potential for actuating servo valves. In Proceedings of the Bath/ASME symposium on fluid power and motion control (Vol. 6).

[17]. Tamburrano, P., Amirante, R., Distaso, E., \& Plummer, A. R. (2018). Full simulation of a piezoelectric double nozzle flapper pilot valve coupled with a main stage spool valve. Energy Procedia, 148, pp. 487-494

[18]. J Persson, L. J., Plummer, A. R., Bowen, C. R., \& Brooks, I. (2015, October). Design and modelling of a novel servovalve actuated by a piezoelectric ring bender. In ASME/BATH 2015 Symposium on fluid power and motion control.

[19]. Tamburrano, P., Amirante, R., Distaso, E., and Plummer, A.R. (2018). A Novel Piezoelectric Double-Flapper Servovalve Pilot Stage: Operating Principle and Performance Prediction. In Bath/ASME Symposium on Fluid Power and Motion Control FPMC 2018, 12 - 14 September 2018, University of Bath, Bath (UK).

[20]. Noliac,

http://www.noliac.com/products/actuators/plates tacks/. Accessed September 2017

[21]. Matlab \& Simulink. SimscapeTM user's guide R2018a. Mathworks.

\section{ACKNOWLEDGMENT}

This research has been supported by the European Commission under the Marie Curie Intra-European fellowship Programme. EC Grant Agreement n. 701336, H2020 MSCA Individual Fellowship: Development of a novel servovalve concept for aircraft (DNSVCFA). Start date: 01/09/2017, End date: 19/10/2019, Location of the project: University of Bath. 